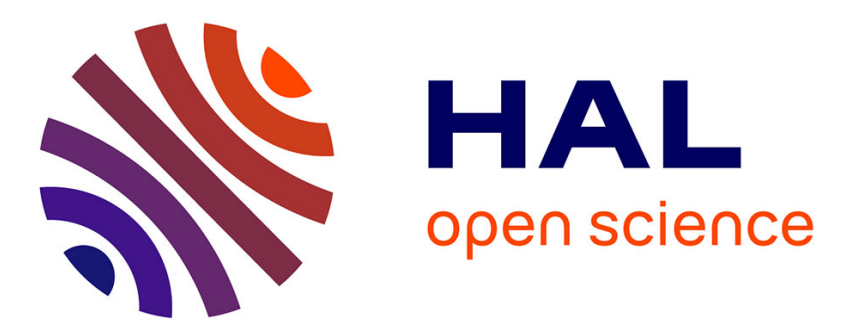

\title{
Destruction par chocs électroniques des atomes métastables et pseudo-métastables de néon dans une colonne positive
}

\author{
J. Leveau, S. Valignat, F. Deigat
}

\section{- To cite this version:}

J. Leveau, S. Valignat, F. Deigat. Destruction par chocs électroniques des atomes métastables et pseudo-métastables de néon dans une colonne positive. Journal de Physique Lettres, 1977, 38 (19), pp.385-388. 10.1051/jphyslet:019770038019038500 . jpa-00231402

\section{HAL Id: jpa-00231402 https://hal.science/jpa-00231402}

Submitted on 1 Jan 1977

HAL is a multi-disciplinary open access archive for the deposit and dissemination of scientific research documents, whether they are published or not. The documents may come from teaching and research institutions in France or abroad, or from public or private research centers.
L'archive ouverte pluridisciplinaire HAL, est destinée au dépôt et à la diffusion de documents scientifiques de niveau recherche, publiés ou non, émanant des établissements d'enseignement et de recherche français ou étrangers, des laboratoires publics ou privés. 


\title{
DESTRUCTION PAR CHOCS ÉLECTRONIQUES DES ATOMES MÉTASTABLES ET PSEUDO-MÉTASTABLES DE NÉON DANS UNE COLONNE POSITIVE
}

\author{
J. LEVEAU, S. VALIGNAT et F. DEIGAT
}

E.R.A. C.N.R.S. $n^{\circ}$ 302, Université Claude-Bernard Lyon I, Laboratoire de Spectroscopie et de Luminescence, 43, bd du 11-Novembre-1918, 69621 Villeurbanne, France

(Reçu le 11 juillet 1977, révisé le 29 août 1977, accepté le 31 août 1977)

Résumé. - La réponse en fréquence de la population des niveaux $1 \mathrm{~s}_{3}, 1 \mathrm{~s}_{4}$ et $1 \mathrm{~s}_{5}$ du néon, dans une colonne positive, à une variation sinusoïdale du courant de décharge a été étudiée. On en déduit les coefficients globaux de désexcitation par chocs électroniques :

$$
\begin{aligned}
& \beta\left(1 \mathrm{~s}_{3}\right)=\beta\left(1 \mathrm{~s}_{5}\right)=(10,4 \pm 2) 10^{-7} \mathrm{~cm}^{3} \cdot \mathrm{s}^{-1} \\
& \beta\left(1 \mathrm{~s}_{4}\right)=(27 \pm 4) 10^{-7} \mathrm{~cm}^{3} \cdot \mathrm{s}^{-1} .
\end{aligned}
$$

Abstract. - The population frequency response of the $1 s_{3}, 1 s_{4}, 1 s_{5}$ levels of Neon to a weak modulation of the discharge current has been studied in the positive column of a glow discharge. The following values are found for the deexcitation coefficients by electron impact :

$$
\begin{aligned}
& \beta\left(1 \mathrm{~s}_{3}\right)=\beta\left(1 \mathrm{~s}_{5}\right)=(10.4 \pm 2) 10^{-7} \mathrm{~cm}^{3} \cdot \mathrm{s}^{-1} \\
& \beta\left(1 \mathrm{~s}_{4}\right)=(27 \pm 4) 10^{-7} \mathrm{~cm}^{3} \cdot \mathrm{s}^{-1} .
\end{aligned}
$$

1. Introduction. - Parmi les processus de destruction par chocs électroniques des niveaux métastables $1 s_{3}$ et $1 s_{5}$, et pseudo-métastables $1 s_{4}$ (Fig. 1) de néon dans une colonne positive, le plus important est celui décrit dans [1] [2] qui intervient dans la formation par étape des niveaux $2 p_{i}$ à partir des niveaux $1 s_{j}$.

Dans ces articles, les auteurs déduisent de la mesure de l'intensité de la lumière de fluorescence émise par chacun des niveaux $2 p$ les coefficients d'excitation $\langle\sigma V\rangle_{\left\{1 \mathrm{~s}_{\mathrm{j}}\right\}-2 \mathrm{p}_{\mathbf{i}}}$ où tous les niveaux $1 \mathrm{~s}_{\mathrm{j}}$ sont considérés comme un niveau unique.

Seuls I. M. Beterov et V. P. Chebotaev, afin de mettre en évidence le rôle joué par chacun des niveaux $1 \mathrm{~s}$, calculent, à partir de ces coefficients, les termes $\langle\sigma V\rangle_{1 s_{j}-2 p_{i}}$ dont ils déduisent les sections de chocs $\sigma_{1 \mathrm{~s}_{\mathrm{j}}-2 \mathrm{p}_{\mathrm{i}}}$.

Le but de ce travail est de faire, sur chacun des niveaux métastables, une mesure directe de sa fréquence de relaxation due aux chocs électroniques. Nous comparerons nos résultats expérimentaux à [2] et à ceux d'un calcul effectué à partir d'expressions théoriques données par H. W. Drawin [3].

La méthode que nous utilisons est la suivante : nous réalisons une faible modulation du courant de décharge, ce qui produit une modulation de la population de tous les niveaux excités. Sous certaines condi-

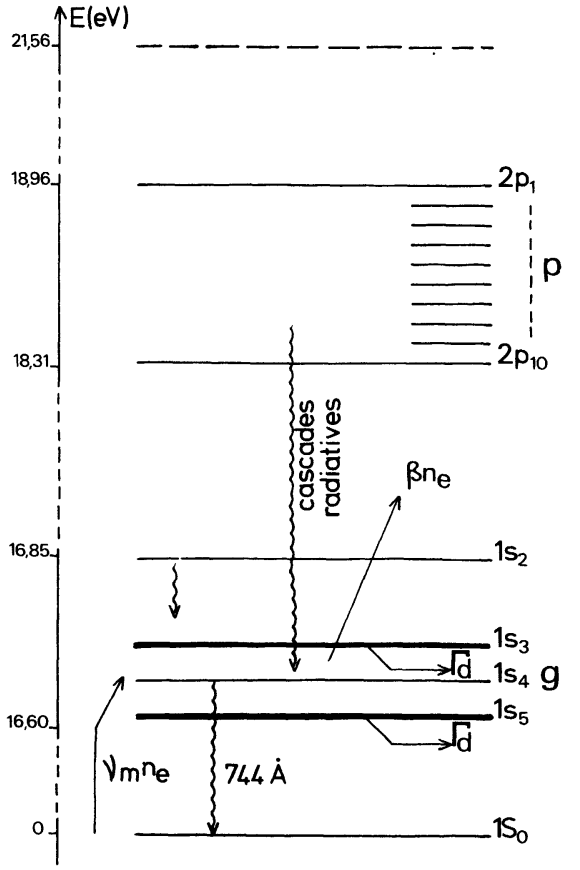

Fig. 1. - Schéma des premiers niveaux excités du néon. On a utilisé la notation de Paschen : $1 \mathrm{~s}_{5}\left({ }^{3} \mathrm{P}_{2}\right) ; 1 \mathrm{~s}_{4}\left({ }^{3} \mathrm{P}_{1}\right) ; 1 \mathrm{~s}_{3}\left({ }^{3} \mathrm{P}_{0}\right)$.

[Scheme of the first excited levels of Neon. Paschen notation is used : $\left.1 \mathrm{~s}_{5}\left({ }^{3} \mathrm{P}_{2}\right) ; 1 \mathrm{~s}_{4}\left({ }^{3} \mathrm{P}_{1}\right) ; 1 \mathrm{~s}_{3}\left({ }^{3} \mathbf{P}_{0}\right) \cdot\right]$ 
tions qui seront précisées plus loin, l'étude de la réponse en fréquence de la population de chacun des niveaux 1s à la modulation du courant de décharge, donne la fréquence de relaxation de ces niveaux.

2. Equations de base. - La densité électronique $n_{\mathrm{e}}$ et la population d'un niveau métastable $N_{\mathrm{g}}$ obéissent aux équations suivantes :

$$
\begin{aligned}
& \frac{\partial n_{\mathrm{e}}}{\partial t}=D_{\mathrm{a}} \Delta n_{\mathrm{e}}+v_{\mathrm{i}} n_{\mathrm{e}}+\sum_{\mathrm{g}} Y N_{\mathrm{g}} n_{\mathrm{e}} \\
& \frac{\partial N_{\mathrm{g}}}{\partial t}=D \Delta N_{\mathrm{g}}+v_{\mathrm{m}} n_{\mathrm{e}}-\beta n_{\mathrm{e}} N_{\mathrm{g}}+\sum_{\mathrm{p}} C_{\mathrm{pg}} N_{\mathrm{p}}
\end{aligned}
$$

Les premiers termes de chacun des seconds membres sont des termes de diffusion. Dans (2) on peut écrire [4]: $D \Delta N_{\mathrm{g}}=-\Gamma_{\mathrm{d}} N_{\mathrm{g}} . \quad v_{\mathrm{i}}$ est la fréquence d'ionisation à partir du niveau fondamental et $Y$ est le taux d'ionisation à partir du niveau métastable. $v_{\mathrm{m}} n_{\mathrm{e}}$ est le nombre d'atomes excités par seconde dans l'état g. Le dernier terme de (2) représente les transferts par cascade radiative à partir des niveaux excités supérieurs. $\beta$ est le taux de désexcitation par collisions électroniques.

Dans le cas où $\sum_{\mathrm{g}} Y N_{\mathrm{g}} n_{\mathrm{e}} \ll v_{\mathrm{i}} n_{\mathrm{e}}$, les éqs. (1) et (2) sont faciles à résoudre. Pour cette dernière, on obtient, en admettant qu'une variation de courant $i_{\mathrm{c}}=i+\delta i \mathrm{e}^{j \omega t}$ conduit à une variation identique de la densité électronique, et en négligeant les termes du second ordre $(\delta i \ll i)$ :

$$
\delta N_{\mathrm{g}}=\frac{\left(v_{\mathrm{m}}-\beta N_{\mathrm{g}}\right) \delta n_{\mathrm{e}}+\delta\left[\sum_{\mathrm{p}} C_{\mathrm{pg}} N_{\mathrm{p}}\right]}{\mathrm{j} \omega+\omega_{\mathrm{c}}}
$$

avec

$$
\omega_{\mathrm{c}}=\Gamma_{\mathrm{d}}+\beta n_{\mathrm{e}} .
$$

Quand le numérateur de cette expression est indépendant de $\omega$, la mesure de $\delta N_{\mathrm{g}}$ en fonction de $\omega$ donne une courbe lorentzienne dont on déduit $\omega_{\mathrm{c}}$ (Fig. 2).

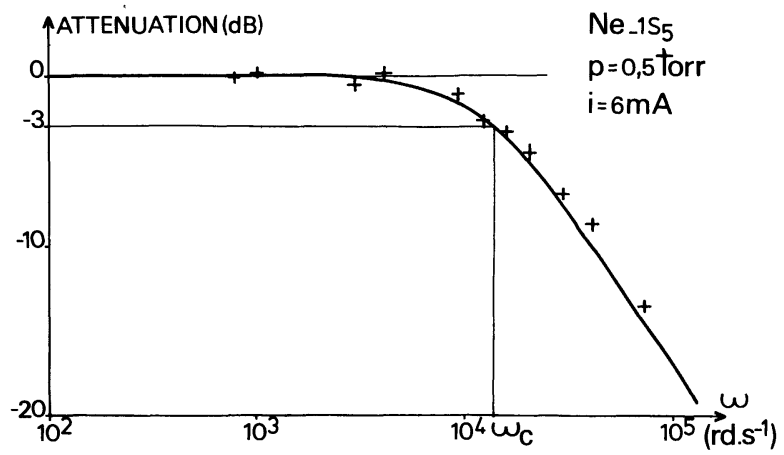

Fig. 2. - Un exemple d'atténuation du coefficient d'absorption en fonction de la fréquence de modulation du courant de décharge. + : points expérimentaux. En trait plein : courbe de Lorentz idéale.

[Example of attenuation of the absorption coefficient versus the modulation frequency of the discharge current. + : experimental. Continuous : ideal lorentzian line.]
Tout écart par rapport à la courbe de Lorentz théorique marque les limites du domaine de validité de notre modèle.

3. Conditions expérimentales et résultats. - La pression dans le tube d'expérience, dont le rayon est $r=5 \mathrm{~mm}$, est $p=0,5$ torr. Dans ces conditions on montre que la condition $v_{\mathrm{i}} \gg r N_{\mathrm{g}}$ est respectée. Aux fréquences que nous avons utilisées

$$
100 \mathrm{~Hz} \leqslant \omega / 2 \pi \leqslant 30 \mathrm{kHz},
$$

on a $\delta n_{\mathrm{e}} / n_{\mathrm{e}} \# \delta i / i[5]$; on peut en outre admettre que le numérateur de (3) est indépendant de $\omega . \delta N_{\mathrm{g}}$ est mesuré par absorption optique à l'aide des raies $5945 \AA$ pour $1 \mathrm{~s}_{5}\left(2 \mathrm{p}_{4}-1 \mathrm{~s}_{5}\right), 6096 \AA$ pour $1 \mathrm{~s}_{4}\left(2 \mathrm{p}_{4}-1 \mathrm{~s}_{4}\right), 6266 \AA$ pour $1 s_{3}\left(2 p_{5}-1 s_{3}\right)$. Pratiquement, nous avons accès au coefficient d'absorption $A=\left(I_{0}-I_{\mathrm{t}}\right) / I_{0}$, mais on peut établir à partir de [6] que la composante à $\omega$ de $A$ est proportionnelle à $\delta N_{\mathrm{g}}$, le facteur de proportionnalité étant indépendant de $\omega$.

Nous avons mesuré la densité électronique par sonde de Langmuir. Les résultats sont en bon accord avec ce que l'on peut déduire des mesures effectuées dans [7]. Il y a proportionnalité entre $i$ et $n_{\mathrm{e}}$ dans le domaine de courant exploré :

$$
n_{\mathrm{e}} / i=0,7 \times 10^{9} \mathrm{~cm}^{-3} \mathrm{~mA}^{-1} .
$$

Sur la figure 3, on observe que pour $i<24 \mathrm{~mA}$ les variations de $\omega_{\mathrm{c}}$ sont linéaires avec le courant. Dans le même domaine, les courbes expérimentales $\delta N_{\mathrm{g}}(\omega)$ sont très proches de la courbe lorentzienne idéale.

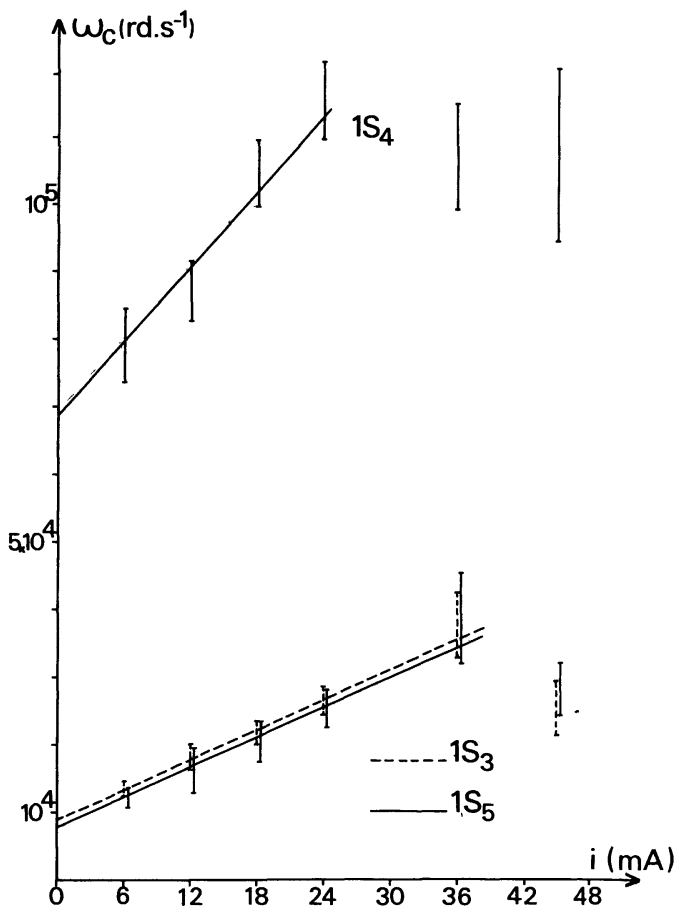

FIG. 3. - Fréquence de désexcitation des niveaux $1 \mathrm{~s}_{3}, 1 \mathrm{~s}_{4}$ et $1 \mathrm{~s}_{5}$ en fonction du courant de décharge.

[Deexcitation frequency of the $1 s_{3}, 1 s_{4}$ and $1 s_{5}$ levels versus discharge current.] 
Au-delà de $i=24 \mathrm{~mA}$, on peut expliquer les saturations observées par le rôle très important joué par les collisions électroniques coupant entre eux les niveaux $1 s_{3}, 1 s_{4}$ et $1 s_{5}$.

On peut constater que les résultats sont très voisins pour les niveaux $1 \mathrm{~s}_{3}$ et $1 \mathrm{~s}_{5}$. Les ordonnées à l'origine donnent :

$$
\begin{aligned}
& \Gamma_{\mathrm{d}}\left(1 \mathrm{~s}_{3}\right)=9300 \pm 1000 \mathrm{~s}^{-1}, \\
& \Gamma_{\mathrm{d}}\left(1 \mathrm{~s}_{5}\right)=8500 \pm 1500 \mathrm{~s}^{-1},
\end{aligned}
$$

ce qui conduit aux coefficients de diffusion

$$
\begin{aligned}
& D_{\mathrm{M}}\left(1 \mathrm{~s}_{3}\right)=195 \pm 20 \mathrm{~cm}^{2} \cdot \mathrm{s}^{-1} \cdot \text { torr }, \\
& D_{\mathrm{M}}\left(1 \mathrm{~s}_{5}\right)=178 \pm 30 \mathrm{~cm}^{2} \cdot \mathrm{s}^{-1} \cdot \text { torr } .
\end{aligned}
$$

Ces résultats sont en très bon accord avec ceux obtenus par A. V. Phelps [8].

Les valeurs du taux de désexcitation par chocs électroniques qu'on déduit de la pente des droites sont les suivantes : $\beta\left(1 \mathrm{~s}_{3}\right)=\beta\left(1 \mathrm{~s}_{5}\right)=(10,4 \pm 2) 10^{-7} \mathrm{~cm}^{3} \cdot \mathrm{s}^{-1}$.

La solution de l'équation de bilan du niveau $1 \mathrm{~s}_{4}$ peut s'écrire sous la même forme que l'éq. (3), avec $\omega_{\mathrm{c}}=\Gamma_{\mathrm{t}}+\beta n_{\mathrm{e}}$, où $\Gamma_{\mathrm{t}}$ qui ne dépend que de la pression est la fréquence de relaxation apparente du niveau, résultant de l'emprisonnement de la raie $\lambda=744 \AA$.

Nous avons $\Gamma_{t}\left(1 \mathrm{~s}_{4}\right)=69000 \pm 6000 \mathrm{~s}^{-1}$, alors que la valeur que l'on peut calculer à partir de la théorie d'Holstein [9] à $p=0,5$ torr est

$$
\Gamma_{\mathrm{t}}\left(1 \mathrm{~s}_{4}\right)=78000 \mathrm{~s}^{-1} \text {. }
$$

Nous obtenons en outre

$$
\beta\left(1 \mathrm{~s}_{4}\right)=(27 \pm 4) 10^{-7} \mathrm{~cm}^{3} \cdot \mathrm{s}^{-1} .
$$

A notre connaissance, la seule valeur de la fréquence de désexcitation par impacts électroniques des niveaux métastables et pseudo-métastables du néon dans une colonne positive résulte d'une estimation faite par A. Ricard [7] qui donne pour le niveau $1 s_{5}$ :

$$
\beta\left(1 \mathrm{~s}_{5}\right)=(3,1 \pm 2) 10^{-7} \mathrm{~cm}^{3} \cdot \mathrm{s}^{-1} .
$$

4. Comparaison des résultats avec [2] et [3]. Comme nous l'avons dit lors de l'introduction, on peut légitimement faire l'hypothèse que dans le domaine où $\omega_{\mathrm{c}}$ varie linéairement avec le courant, la destruction des niveaux $1 s_{j}$ par chocs électroniques se fait principalement suivant la réaction :

$$
\mathrm{Ne}\left(1 \mathrm{~s}_{\mathbf{j}}\right)+\overline{\mathrm{e}} \rightarrow \mathrm{Ne}\left(2 \mathrm{p}_{\mathrm{i}}\right)+\overline{\mathrm{e}} .
$$

Aussi, nous avons porté dans le tableau I les valeurs de la section de choc correspondant à chacune des transitions de ce type, tirées de [2] et [3]. Etant donné que les différences d'énergie $E_{k}=E\left(1 \mathrm{~s}_{\mathrm{j}}\right)-E\left(2 \mathrm{p}_{\mathrm{i}}\right)$ restent très voisines $\left(E_{k} \# 2 \mathrm{eV}\right)$, on peut, par simple addition, déduire la valeur de la section de choc $\sigma_{1 s_{j}-\left\{2 p_{i}\right\}}$ reliant un niveau $1 s_{j}$ à l'ensemble des niveaux $2 p_{i}$ (avant-dernière colonne).

Avec la même hypothèse que ci-dessus, nous pouvons déduire des valeurs de $\beta$ les mêmes sections de choc (dernière colonne). Le calcul est facile en supposant une répartition Maxwellienne de la vitesse des électrons, et en admettant que :

$$
\begin{array}{lll}
\sigma(E)=0 & \text { pour } & E<E_{k} \\
\sigma(E)=\sigma^{\max } & \text { pour } & E>E_{k}
\end{array}
$$

on a alors :

$$
\begin{aligned}
\beta_{1 \mathrm{~s}_{\mathrm{j}}} \# & \langle\sigma V\rangle_{1 \mathrm{~s}_{\mathrm{j}}-\left\{2 \mathrm{p}_{\mathrm{i}}\right\}}= \\
& =\sigma_{1 \mathrm{~s}_{\mathrm{j}}-\left\{2 \mathrm{p}_{\mathrm{i}}\right\}}^{\max } \bar{V}_{\mathrm{e}}\left(1+\frac{E_{k}}{k T e}\right) \exp \left(-\frac{E_{k}}{k T e}\right)
\end{aligned}
$$

Nous avons mesuré par sonde de Langmuir: $k T e=5,9 \mathrm{eV}$.

Ces valeurs sont en bon accord avec les résultats de I. M. Beterov et V. P. Chebotaev, ce qui confirme l'importance du seul processus de destruction que nous avons envisagé ici. Toutefois, les écarts observés ne nous permettent pas de tirer de plus amples conclusions, étant donnée l'erreur très importante attachée à ces derniers résultats.

Si nous comparons maintenant les sections de chocs que nous obtenons à celles déduites de [3], on constate que nos valeurs sont supérieures (surtout pour le

\section{TABLEAU I}

Section de choc $\sigma_{1 \mathrm{~s}_{\mathrm{j}}-2 \mathrm{p}_{\mathrm{i}}}\left(\right.$ en $\left.10^{-15} \mathrm{~cm}^{2}\right)$. Ligne du haut [2]. Ligne du bas [3] : les forces d'oscillateur ont été prises dans [10]. Le calcul n'est possible que pour les transitions optiques permises.

[Cross-section $\sigma_{1 \mathrm{~s}_{1}-2 \mathrm{p}_{\mathrm{i}}}\left(\right.$ in $10^{-15} \mathrm{~cm}^{2}$ ). Upper line [2]. Lower line [3] :

oscillator strengths are given by [10]. Calculations are only possible for the optically allowed transitions.]

\begin{tabular}{lllllllllllll} 
& $2 \mathrm{p}_{1}$ & $2 \mathrm{p}_{2}$ & $2 \mathrm{p}_{3}$ & $2 \mathrm{p}_{4}$ & $2 \mathrm{p}_{5}$ & $2 \mathrm{p}_{6}$ & $2 \mathrm{p}_{7}$ & $2 \mathrm{p}_{8}$ & $2 \mathrm{p}_{9}$ & $2 \mathrm{p}_{10}$ & \multicolumn{2}{c}{$2 \mathrm{p}_{\mathrm{i}}$} \\
& 2,1 & & & 4,2 & & 2,4 & & & & 8,7 & 6,7 \\
$1 \mathrm{~s}_{3}$ & 1,3 & & & 2 & & 1,4 & & & 0,6 & 5,3 & \\
& 0,3 & 0,9 & 5,7 & 0,2 & 1,1 & 1,4 & 4 & & 1,9 & 15,5 & \\
$1 \mathrm{~s}_{4}$ & 0,2 & 0,5 & 0,7 & 0,1 & 0,3 & 0,9 & 1,3 & & 0,6 & 4,6 & 17,4 \\
& & 0,4 & & 1,1 & 0,2 & 2,2 & 0,3 & 1,3 & 4,8 & 1,8 & 12,1 & \\
$1 \mathrm{~s}_{5}$ & 0,2 & & 0,3 & & 0,6 & 0,1 & 0,4 & 1,9 & 0,6 & 4,1 & 6,7
\end{tabular}


niveau $1 s_{4}$ ), ce qui permet de penser que les autres processus de destruction peuvent jouer un rôle non négligeable. Citons parmi eux les chocs ionisants, et surtout les chocs avec les électrons qui couplent entre eux les niveaux métastables.

Remarquons pour conclure les écarts, importants pour certaines transitions, qu'il y a entre les sections de chocs tirées de [2] et [3]. Cela souligne l'intérêt qu'il peut y avoir à une mesure directe des coefficients $\langle\sigma V\rangle_{1 \mathrm{~s}_{\mathrm{j}}-2 \mathrm{p}_{\mathrm{i}}}$.

Nous pensons parvenir prochainement à un tel résultat, grâce aux expériences actuellement en cours, qui utilisent pour la modulation des populations la lumière issue d'un laser accordable, ce qui permet alors une analyse détaillée des différents processus de désexcitation.

\section{Bibliographie}

[1] Frish, S. E. et Revald, V. F., Opt. Spectrosc. 15 (1963) 395. [2] Beterov, I. M. et Chebotaev, V. P., Opt. Spectrosc. 23 (1967) 467.

[3] Drawin, H. W., Rapport EUR-C.E.A.-F.C.-383, révisé 1967 Association Euratom-C.E.A., Fontenay-aux-roses (1966).

[4] Delcroix, J. L., Physique des plasmas (Dunod) 1966, Tome 2.

[5] Davy, P., J. Physique Colloq. 29 (1968) C3-224.

[6] Mitchell, A. G. C. et Zemansky, M. W., Resonance Radiation and Excited Atoms (The Mac Millan Company, New York) 1934.

[7] RicARD, A., Thèse no 431, Toulouse 1971.

[8] Phelps, A. V., Phys. Rev. 114 (1959) 1011.

[9] Holstein, T., Phys. Rev. 83 (1951) 1159.

[10] Wiese, W. L., Smith, M. W., Glennon, B. M., Atomic transition Probabilities (N.S.R.D.S., N.B.S.) 4 (1966), vol. 1. 